\title{
Effect of nanocrystals concentration on optical and luminescent properties of PVK:ZnSe nanocomposites
}

\author{
KANUPRIYa Sharma $^{1,2}$, Praveen Kumar ${ }^{1, *}$, GaUraV Verma $^{2,3}$ \\ ${ }^{1}$ Department of Physics, DAV University, Sarmastpur, Jalandhar-144012, India \\ ${ }^{2}$ Dr. Shanti Swarup Bhatnagar University Institute of Chemical Engineering and Technology, Panjab University, \\ Chandigarh-160014, India \\ ${ }^{3}$ Center for Nanoscience \& Nanotechnology, UIEAST, Panjab University, Chandigarh-160014, India
}

\begin{abstract}
This work presents a systematic study of the effect of ZnSe nanocrystals (NCs) concentration on the optical and luminescent properties of poly N-vinylcarbazole (PVK) polymer nanocomposites. The ZnSe nanocrystals were synthesized by a simple coprecipitation chemical route, while PVK:ZnSe nanocomposite films were fabricated using the spin coating technique. The samples were characterized by XRD, TEM, SEM, UV-Vis and fluorescence techniques. The X-ray diffraction and TEM studies confirmed the particle size, microstructure and spherical shape of the synthesized nanocrystals. The ZnSe nanocrystals in PVK caused a decrease in optical gap with increasing concentration of nanocrystals. The emission spectra exhibited augmentation in intensity up to $70 \mathrm{wt} . \%$ of nanoparticles while further addition resulted in a decrease in luminescence. The structure-property relationships obtained for the present system are important for developing low cost illumination devices.
\end{abstract}

Keywords: nanocrystals; PVK:ZnSe nanocomposites; XRD; optical and fluorescence properties

\section{Introduction}

Over the past years, organic light-emitting diodes (OLED) incorporating polymer matrices and inorganic nanocrystals have been developing very quickly for their potential application in flat panel displays. The use of semiconductor nanocrystals (NCs) received strong attention because of quantum confinement effects [1], imparting size tunable optical and electronic properties important for light-emitting diodes [2], photovoltaic devices [3], etc. The $\mathrm{ZnSe}$ semiconductor has a direct and wide-band gap $(2.7 \mathrm{eV})$, and a large exciton binding energy $(21 \mathrm{meV})$ leading to interesting physical properties. It is a good candidate for being used in light emitting devices and laser diodes. Many synthetic methods have been developed for preparing these materials, including photochemical method, sonochemical method [4], hydrothermal method [5], microwave heating process [6], microemulsion method [7], etc.

*E-mail: prafiziks@gmail.com
The fabrication of hybrid organic-inorganic materials favors the enhancement in the carrier injection and recombination processes which are very important for realization energy efficient and highly luminescent light emitting devices [8]. The synthesis of highly dispersed inorganic nanomaterials in transparent organic polymer matrices has a great advantage of easy fabrication and enhanced materials properties [9]. One of the most widely studied polymers is PVK which shows a good combination of electrical and optical properties making it a good candidate for optoelectronic applications such as light emitting diodes [10]. The resonant transfer of energy through formation of hybrid excitons is very sensitive to the external perturbations or varying in weight percentages in the heterostructure [11]. The most important mechanism is the exciton capture by NPs in a polymer matrix [12].

The capping of nanoparticles with polymers was found to increase the surface stability along with significant influence on the morphology and optical properties of nanocomposites [13]. However, there are only few reports 
on the influence of different concentrations on the characteristics of polymer based organic/inorganic hybrid nanocomposites. The nanocomposite PVK:ZnSe presents a promising area in the LEDs application as shown by Mastour et al. [14]. The increase in weight percentage leads to an increase in the fluorescence intensity. This result shows the importance of studying the optical absorption spectra of these nanocomposites in order to deduce the band gap energy.

In this paper, we report the effect of $\mathrm{ZnSe}$ nanocrystals concentration on the optical and luminescence properties of spin coated PVK:ZnSe nanocomposites. The enhancement in the luminescence with the concentration has been reported for the PVK:ZnSe nanocomposites.

\section{Experimental}

\subsection{Materials}

ZnSe nanoparticles were prepared by simple co-precipitation method in aqueous medium. All the chemicals: zinc chloride (Aldrich, Fisher), selenium powder (Loba Chemie), sodium sulfate (CDH), PVK (Aldrich) were of analytical grade and used as purchased without further purification.

\subsection{Synthesis of $\mathrm{ZnSe}$ nanoparticles}

The sodium selenosulfate was prepared by dissolving $5 \mathrm{mM}$ selenium powder and $30 \mathrm{mM}$ sodium sulfate in $100 \mathrm{~mL}$ distilled water to get sodium selenosulfate solution. The solution was continuously stirred for $8 \mathrm{~h}$, kept overnight and finally filtered to get a clear solution of sodium selenosulfate for further reactions. For the synthesis of $\mathrm{ZnSe}$ nanoparticles, $0.1 \mathrm{M}$ zinc chloride was dissolved in $100 \mathrm{~mL}$ distilled water and then sodium selenosulfate solution was added dropwise along with $1 \mathrm{~mL}$ of hydrazine hydrate as a stabilizer. Few drops of ammonia solution were added to get a transparent mixture. $1 \mathrm{M} \mathrm{NaOH}$ solution was further added into the mixtures to adjust the $\mathrm{pH}$ and to achieve precipitation of ZnSe nanoparticles [15]. Finally, the product was separated by centrifugation and washed with distilled water and ethanol several times.
The nanoparticles were finally dried in a hot air oven at $50{ }^{\circ} \mathrm{C}$ overnight to get a powdered sample.

\subsection{PVK:ZnSe nanocomposite films}

For the fabrication of PVK:ZnSe nanocomposites, PVK was dissolved in chloroform $(2 \mathrm{mg} / \mathrm{mL})$ and different concentrations of $\mathrm{ZnSe}$ nanoparticles (0 wt.\%, 10 wt.\%, 30 wt. $\%, 50$ wt.\%, 70 wt.\% and 90 wt.\%) were added to this solution and stirred at room temperature for $2 \mathrm{~h}$. The solution was then coated on the well cleaned glass substrates $(2000 \mathrm{rpm}$ for $60 \mathrm{~s}$ ) by using spin coating unit (Model: spin NXG-P1, Apex instruments, Kolkata). Finally, the samples were dried at $100{ }^{\circ} \mathrm{C}$ in a hot air oven.

\subsection{Samples characterization}

The synthesized ZnSe nanocrystals were characterized by X-ray diffractometer (Model: X'Pert PRO, PANalytical, UK) equipped with a goniometer (PW3050/60) using $\mathrm{CuK} \alpha$ radiation $(\lambda=1.5406 \AA)$ in the $2 \theta$ range of $20^{\circ}$ to $80^{\circ}$. The shape and microstructure of ZnSe nanocrystals were determined using transmission electron microscope (Model: JEM-2100, JEOL). Surface morphology of the nanocomposite films was analyzed using scanning electron microscope (Model: Quanta 200 F SEM, FEI, USA). The infrared spectrum of the nanoparticles was recorded on the FT-IR spectrophotometer (Model: RZX, PerkinElmer, USA). The absorption spectra of colloidal nanoparticles and nanocomposite films were recorded by UV-Vis spectrophotometer (Lambda750, PerkinElmer). The emission spectra were recorded using a fluorescence spectrometer with a xenon flash lamp as excitation source (Model: Cary Eclipse, Agilent, USA).

\section{Results and discussion}

\subsection{Characterization of ZnSe nanocrys- tals}

Fig. 1a shows the X-ray diffraction pattern of pure $\mathrm{ZnSe}$ nanocrystals. The diffraction peaks at the $2 \theta$ angles of $29.57^{\circ}, 45.23^{\circ}, 56.08^{\circ}$ and $61.42^{\circ}$ correspond to the (llll $\left.\begin{array}{lll}1 & 1\end{array}\right),\left(\begin{array}{lll}1 & 1 & 0\end{array}\right),\left(\begin{array}{lll}0 & 0 & 4\end{array}\right)$ 


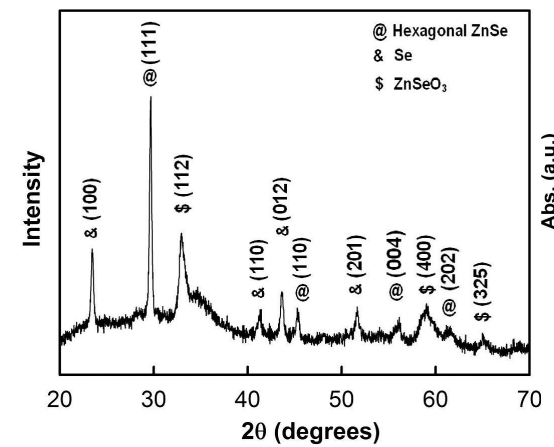

(a)

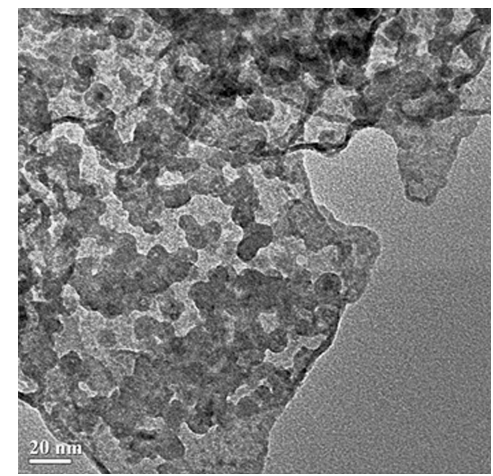

(d)

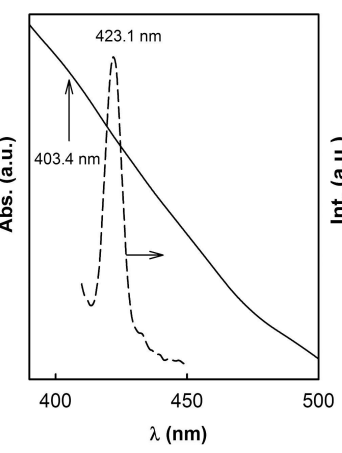

(b)

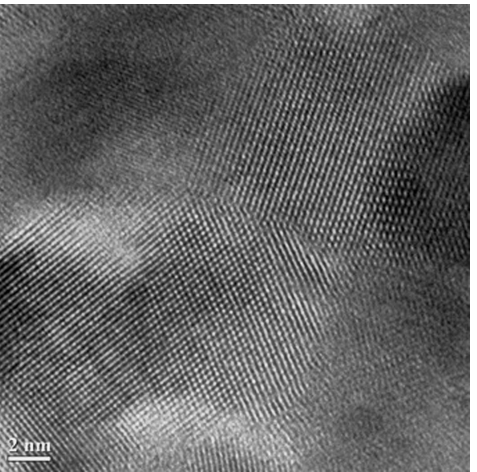

(e)

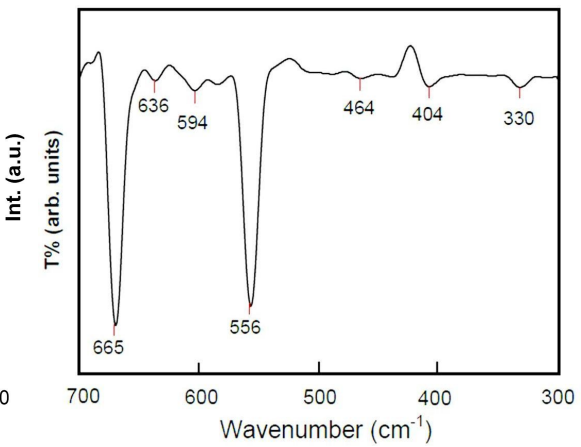

(c)

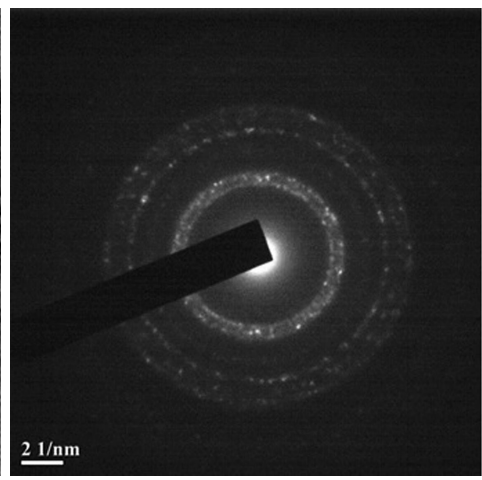

(f)

Fig. 1. (a) XRD pattern, (b) UV-Vis absorption and photoluminescence emission at $370 \mathrm{~nm}$ excitation energy, (c) FT-IR transmission spectrum, (d) TEM image (e) HRTEM image and (f) SAED pattern of the synthesized ZnSe nanoparticles.

and (2 0 2) planes of hexagonal structure of $\mathrm{ZnSe}$ (JCPDS Card No. 80-0008), $23.43^{\circ}, 41.31^{\circ}, 43.54^{\circ}$ and $51.58^{\circ}$ peaks can be assigned to $\left(\begin{array}{lll}1 & 0 & 0\end{array}\right)$, $\left(\begin{array}{lll}1 & 1 & 0\end{array}\right),\left(\begin{array}{lll}0 & 1 & 2\end{array}\right)$ and $\left(\begin{array}{lll}2 & 0 & 1\end{array}\right)$ planes of Se

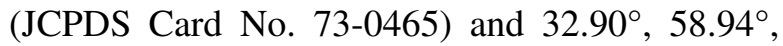
and $64.92^{\circ}$ correspond to ( $\left.\begin{array}{lll}1 & 1 & 2\end{array}\right),\left(\begin{array}{lll}4 & 0 & 0\end{array}\right)$ and (3 2 5) planes of orthorhombic $\mathrm{ZnSeO}_{3}$ (JCPDS Card No. 78-0446) [16, 17]. The formation of other phases may be due to the use of excess sodium selenosulfate along with the atmospheric air during the synthesis process. The crystallite size D was calculated using the Debye-Scherrer formula:

$$
D=0.9 \lambda / \beta \cos \theta
$$

where $\lambda$ is the wavelength of radiation used, $\beta$ is the full width at half maximum (FWHM) of diffraction peak, and $\theta$ is the Bragg angle. The crystallite size calculated from the (lllll $\left.\begin{array}{lll}0 & 1\end{array}\right)$ plane of the $\mathrm{ZnSe}$ nanocrystals was found to be about
$4.63 \mathrm{~nm}$. The presence of a relatively sharp peak in a background of a wider peak suggests that both large and small grains were formed together.

Fig. 1b shows the optical absorption and luminescence spectra of the colloidal $\mathrm{ZnSe}$ nanoparticles [18]. It is observed that the absorption spectrum exhibits a peak at $403.4 \mathrm{~nm}(3.07 \mathrm{eV})$ and the blue shift from the bulk optical gap $(2.70 \mathrm{eV})$ of the sample revealing the formation of $\mathrm{ZnSe}$ nanoparticles. The luminescence spectrum exhibits a strong peak at around $423.1 \mathrm{~nm}$ at the excitation energy $370 \mathrm{~nm}$. Fig. 1c shows the IR transmission spectrum of the $\mathrm{ZnSe}$ nanocrystal in the frequency range of $300 \mathrm{~cm}^{-1}$ to $700 \mathrm{~cm}^{-1}$. The main characteristic absorption peaks at $556 \mathrm{~cm}^{-1}$ and $665 \mathrm{~cm}^{-1}$ are observed and can be ascribed to stretching mode vibrations of $\mathrm{Zn}-\mathrm{Se}$ bonds in $\mathrm{ZnSe}$ [19]. 
Fig. 1d shows the TEM micrograph of the colloidal $\mathrm{ZnSe}$ nanoparticles. This shows the particles are of spherical shape with an average size of $7.3 \mathrm{~nm}$ which is larger than that calculated from Scherrer method. Fig. 1e shows the HRTEM micrograph and revels the interplanar spacing of $0.269 \mathrm{~nm}$ corresponding to $(200)$ plane of $\mathrm{ZnSe}$ nanocrystals. Fig. If shows the selected area electron diffraction (SAED) pattern, exhibiting broad diffused rings corresponding to (1 111$),\left(\begin{array}{lll}2 & 2 & 0\end{array}\right)$ and (3 11 1) planes that are typical of nanosized $\mathrm{ZnSe}$ particles with a cubic structure [20]. Factors, such as supersaturation, nucleation and growth rates, colloidal stability, recrystallization and aging processes, have considerable effect on the particle size and microstructure [21].

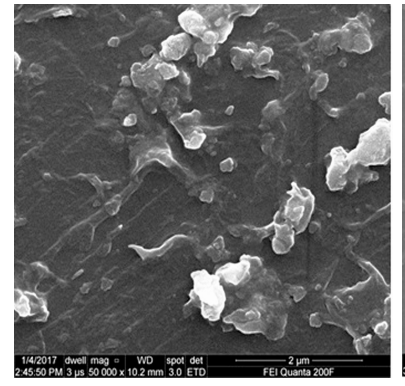

(a)

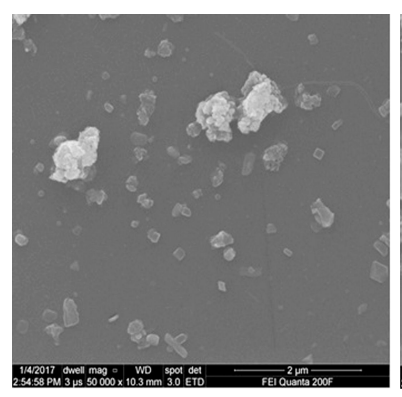

(c)

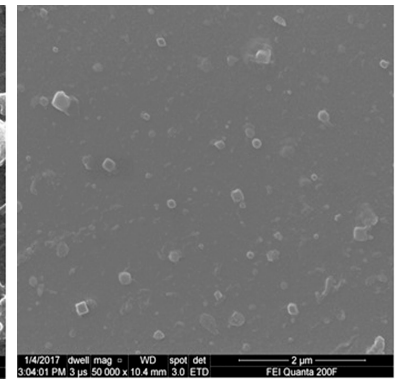

(b)

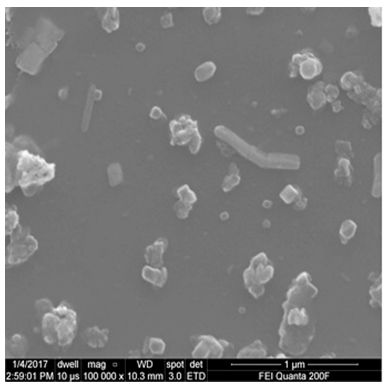

(d)
Fig. 2. FE-SEM images of spin coated (a) pure PVK, and (b) $30 \%$, (c) and (d) $70 \%$ ZnSe nanoparticles in PVK:ZnSe films (various magnifications).

\subsection{Characterization of PVK:ZnSe nanocomposites}

The morphology of prepared nanoparticles was studied by FE-SEM. Fig. 2a to Fig. 2d show the typical FE-SEM images of PVK polymer and PVK:ZnSe nanocomposites. Fig. 2a reveals the irregular shaped surface or porous structure resulting in higher surface roughness of the spin coated polymer films. The incorporation of nanoparticles resulted in an increase in surface smoothness with the emergence of the cubic shaped polymer clusters (the average dimensions are less than $\sim 4 \mathrm{~nm}$ ) in the PVK:ZnSe nanocomposite film as shown in Fig. 2b [22]. However, the increase in $\mathrm{ZnSe}$ nanoparticle concentration favored the aggregation of the cube shaped structures as shown in Fig. 2c, while the enlarged image (Fig. 2d) reveals the cuboid shaped polymer structures on the surface of the films. These structures are typical of the polymer nanocomposites as reported in the literature [23].

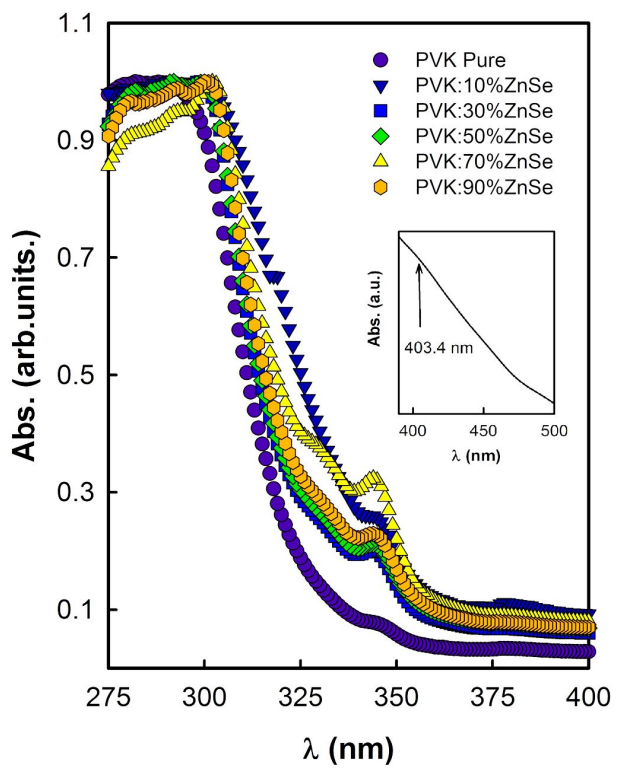

Fig. 3. Optical absorption spectra of spin coated PVK: ZnSe nanocomposite films (inset shows the absorption spectra for $\mathrm{ZnSe}$ nanoparticles).

Fig. 3 shows the optical absorption spectra of the PVK:ZnSe nanocomposites with different concentrations of $\mathrm{ZnSe}$ nanoparticles (inset shows the spectra for $\mathrm{ZnSe}$ nanoparticles). The sharp absorption edge $(298 \mathrm{~nm})$ accompanied with a small bump (345 nm) is attributed to $\mathrm{n} \rightarrow \pi^{*}$ optical transitions in the pure PVK films [24]. The incorporation of small amount of $\mathrm{ZnSe}$ nanoparticles $(10 \mathrm{wt} . \%)$ resulted in a red shift 
and decrease in steepness of absorption edge (304 nm) along with an increase in the absorbance of the bump in PVK:ZnSe nanocomposites. Similar values of the optical gap and red shift in the optical gap have been reported for the PVK:ZnSe composite films [25]. The anomalous variation in the absorption coefficient below the absorption edge can be ascribed to the combined effect of light scattering from the nanoparticles and their aggregated structures formed in the nanocomposite films. Further addition of nanoparticles resulted in an increase in absorption of the shoulder band along with anomalous variation in the absorption edge for the nanocomposites. These results suggest that the incorporation of ZnSe nanoparticles in PVK favored the enhancement in the intensity for $n \rightarrow \pi^{*}$ optical transitions characteristic of the change in the stacking of polymeric units in the PVK:ZnSe nanocomposites.

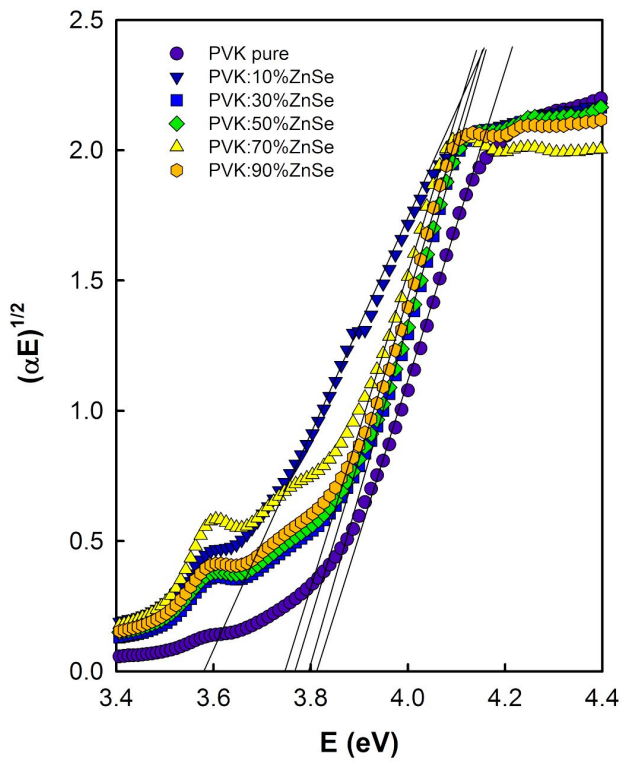

Fig. 4. Plot of $(\alpha E)^{1 / 2}$ vs. photon energy hv for PVK: $\mathrm{ZnSe}$ nanocomposite films.

The absorption coefficient in the high absorption region can be related to the incident photon energy by [22]:

$$
\alpha E=B\left(h v-E_{o}\right)^{2}
$$

where $\mathrm{B}$ is the quality factor of which the inverse $\mathrm{B}^{-1}$ represents the width of band tailing, $\mathrm{h} v$ is

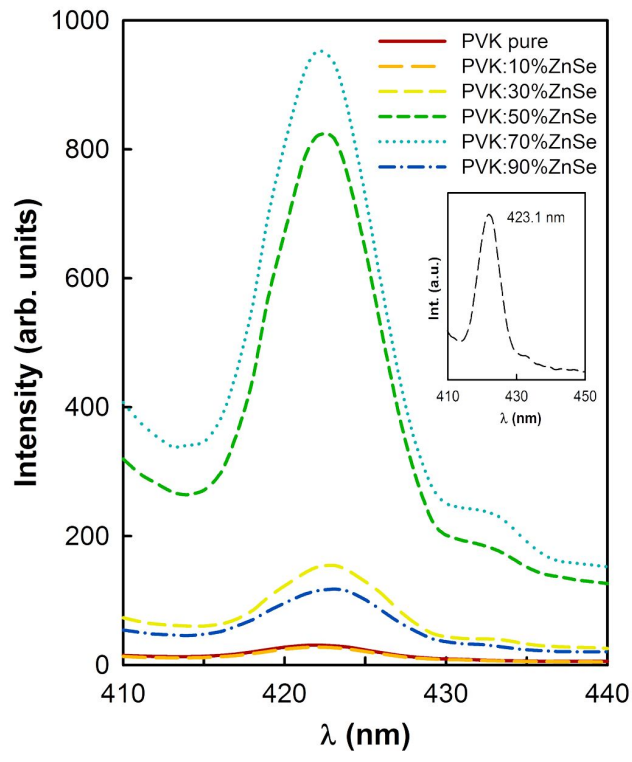

Fig. 5. Emission spectra of PVK:ZnSe nanocomposite films at $400 \mathrm{~nm}$ excitation energy (inset shows the emission spectra of $\mathrm{ZnSe}$ nanoparticles at $370 \mathrm{~nm}$ excitation).

the photon energy and $E_{o}$ is the indirect optical gap. Fig. 4 shows the Tauc plot, i.e. $(\alpha \mathrm{E})^{1 / 2}$ versus photon energy hv for the PVK:ZnSe nanocomposite films with different concentrations of nanoparticles. The optical gap of spin coated PVK film is $3.81 \mathrm{eV}$ which is in a good agreement with the values reported in the literature. The band gap decreases to $3.59 \mathrm{eV}$ with the incorporation of small concentration of $\mathrm{ZnSe}$ nanoparticles $(10 \%)$ in the PVK matrix. This change can be ascribed to large structural changes in the polymer network with nanoparticle addition. Further increase in the nanoparticle concentration resulted in an anomalous variation in the optical gap of the PVK: $\mathrm{ZnSe}$ nanocomposite films. Different types of modification of the polymer network, cluster elimination and their further formation were observed with changing nanoparticle concentration.

Fig. 5 shows the photoluminescence spectra of pure PVK and PVK:ZnSe nanocomposites with different concentrations of $\mathrm{ZnSe}$ nanoparticles $(0 \%$ to $90 \%)$ at $400 \mathrm{~nm}$ excitation (the inset shows the luminescence spectra of $\mathrm{ZnSe}$ nanoparticles at $370 \mathrm{~nm}$ excitation). The spectra exhibit 
two strong emission peaks centered at $420 \mathrm{~nm}$ and $423 \mathrm{~nm}$, accompanied with a shoulder peak at $432 \mathrm{~nm}$ and attributed to the triplet-singlet transitions in pure spin coated PVK films [26]. It has been observed that the addition of nanoparticles $(\mathrm{NP}-\mathrm{x}(\mathrm{x}=10 \%$ to $90 \%))$ resulted in an enhancement in emission peak at $423 \mathrm{~nm}$ as well as in the $432 \mathrm{~nm}$ band (pure PVK) of the hybrid nanocomposites. The luminescence peaks are assigned to PVK as the excitation energy is lower that used for the excitation of $\mathrm{ZnSe}$ nanoparticles. No new emission peaks have been observed in the luminescence spectra of this system, however, the new peaks related to nanoparticles in $\mathrm{Cu}_{2} \mathrm{Se} / \mathrm{PVK}$ system were reported by Govindraju et al. [27]. The luminescence has been found to increase up to 70 wt. $\%$ of $\mathrm{ZnSe}$ nanoparticles addition in the nanocomposites, while further addition of the nanoparticles resulted in some decrease in the luminescence intensity. The enhancement in the intensity can be ascribed to the formation of good quality nanocomposite blends and surface passivation effect of PVK on the $\mathrm{ZnSe}$ nanoparticles in these hybrid materials. However, the decrease in the intensity may be due to the formation of polymer clusters or aggregates in the polymer nanocomposite. Similar results have been reported earlier for such systems [24].

\section{Conclusions}

The effect of concentration of ZnSe nanocrystals on the optical and fluorescence properties of spin coated PVK nanocomposite films has been studied. The analysis of structure and optical properties confirmed the formation of spherical shaped $\mathrm{ZnSe}$ nanocrystals in the quantum confinement regime. The optical gap was found to decrease from $3.81 \mathrm{eV}$ to $3.59 \mathrm{eV}$ with the addition of $10 \% \mathrm{ZnSe}$ nanoparticles while anomalous variation in the optical properties has been observed for PVK:ZnSe nanocomposite films. The optical spectra exhibited a strong emission centered at $423 \mathrm{~nm}$ along with a shoulder peak at $432 \mathrm{~nm}$ for pure spin coated PVK films. The initial increase in concentration of $\mathrm{ZnSe}$ nanocrystals (up to $70 \%$ ) was found to increase the luminescence of PVK but it was found to decrease with further addition of the nanocrystals. The charge transfer process and the aggregation of nanocrystals in the nanocomposites at higher concentrations have been used to explain the variation of fluorescence intensity of the synthesized nanocomposites. The obtained results are very important for designing new organic/inorganic hybrid materials for low cost illumination devices.

\section{Acknowledgements}

One of the authors (P.K.) gratefully acknowledges the financial support from the SERB, DST, New Delhi, India, under the reference No.: FTP/PS-062/2012. The authors are also thankful to the SAIF/CIL, PU, Chandigarh, for XRD; the SMITA Lab, IIT, Delhi, for FESEM, and the NEHU, Meghalaya, for TEM microscopy studies.

\section{References}

[1] Molaei M., Bahador A.R., Karimipour M., J. Lumin., 166 (2015), 101.

[2] Tang A., Teng F., Hou Y., Appl. Phys. Lett., 96 (2010), 163112.

[3] Huynh W.U., Dittmer J.J., Alivisatos A.P., Science 295 (2002), 2425.

[4] Zhu J., Koltypin Y., Gedanken A., Chem. Mater, 12 (2000), 73.

[5] Mollaamin F., Gharibe S., Monajuemi M., Int. J. Phys. Sci., 6 (2011), 1.

[6] Shakir M., Kushwaha S.K., Maurya K.K., Solid State Commun., 149 (2009), 2047.

[7] Hao E., Sun H., Zhou Z., Chem. Mater, 11 (11) (1999), 3096.

[8] Yu W., Xu Z., Teng F., Phys. Lett. A, 338 (2005), 402.

[9] Hariharan P.S., Subhashini N., VasanthalaKSHMI J., J. Fluoresc. 26 (2016), 703.

[10] Benchaabane A., Ben H.Z., Telfah A., Mater. Sci. Semicond. Proc., 64 (2017), 115.

[11] Benchaabane A., Ben H.Z., Sanhoury M.A., Appl. Phys. A, 122 (2016), 1.

[12] Loubiri D., Ben H.Z., Ilahi S., Appl. Phys. A, 119 (2015), 581.

[13] Ben H.Z., Benchaabane A., Kouki F., Synth. Met., 195 (2014), 102.

[14] Mastour N., Ben H.Z., Benchaabane A., Org. Electron., 14 (2013), 2093.

[15] Bakiyaraj G., Dhanasekaran R., Appl. Nanosci., 3 (2013), 125.

[16] Archana J., Navaneethan M., Prakash T., Mater. Lett., 100 (2013), 54.

[17] Zhang B., Ye X., Wang C., J. Mater. Chem., 17 (2007), 2706.

[18] Singh N., Salam Z., Sivasankar N., Mater. Sci. Semicond. Proc., 64 (2017), 16.

[19] Sangareswari M., Sundaram M.M., J. Nanosci. Technol., 1 (2015), 9. 
[20] Baruwati B., Kumar D.K., Manorama S.V., Sensor. Actuat. B-Chem, 119 (2006), 676.

[21] Heiba Z.K., Mohamed M.B., Imam N.G., J. Mol. Struct., 1094 (2015), 91.

[22] Shikha D., Mehta V., Sharma J., J. Mater. Sci.Mater. El., 28 (2017), 8359.

[23] Sharma K., Kumar P., Mater. Focus, 6 (6) (2017), 652

[24] Azizi S., Balhaj M., Zargouni S., Dridi C., Appl. Phys. A-Mater, 123 (2017), 475

[25] Benchaabane A., Hamed Z.B., Kouki F., SANHOURY M.A., Zellama K., Zeinert A., Bouchriha H., J. Appl. Phys., 115 (2014), 134313
[26] Wenge Y., Zheng X., Feng T., Displays, 25 (2004), 61.

[27] Govindraju S., Ntholeng N., Ranganathan K., Moloto M.J., Sikhwivhilu L.M., Moloto N., J. Nanomater., 2016 (2016) 9592189.

Received 2017-10-07 Accepted 2018-05-21 